\title{
Mortality in combined pulmonary fibrosis and emphysema patients is determined by the sum of pulmonary fibrosis and
} emphysema

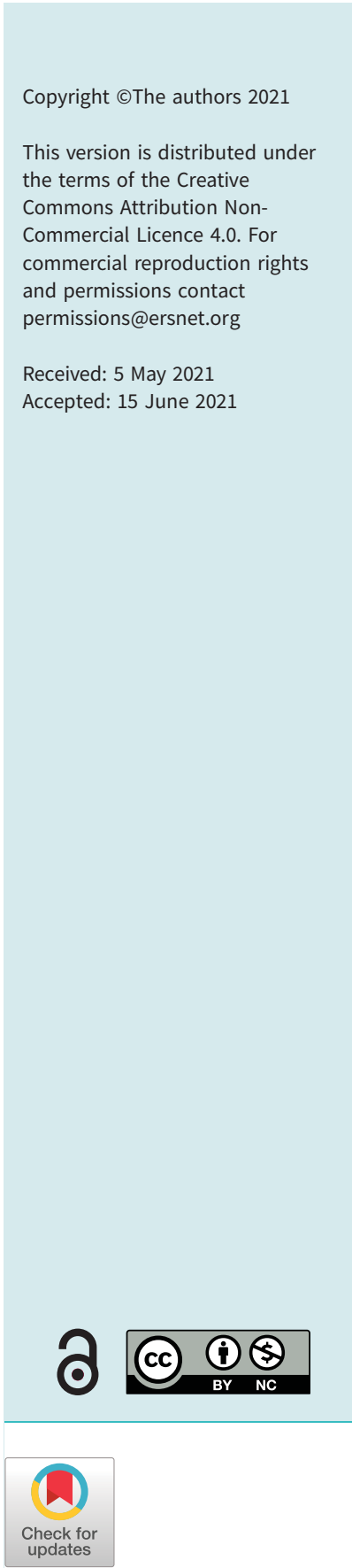

To the Editor:

Emphysema is one of the most common pulmonary comorbidities of idiopathic pulmonary fibrosis (IPF), presenting in about one-third of IPF patients [1]. The term combined pulmonary fibrosis and emphysema (CPFE) has been used to describe a potential phenotype characterised by the coexistence of upper lobe-predominant emphysema, lower lobe-predominant fibrosis and relative preservation of lung volumes (forced vital capacity; FVC) in the context of a disproportionately reduced gas transfer (diffusing capacity of the lung for carbon monoxide; $D_{\mathrm{LCO}}$ ) [1-3]. With regard to patient survival, it remains unclear whether mortality in patients with CPFE reflects the cumulative effects of two disease processes (emphysema and fibrosis), or whether CPFE represents a distinct disease phenotype where outcome is worse than the sum of disease parts (emphysema and fibrosis).

In a previous single centre study [4], we demonstrated that the CPFE phenotype (defined as the presence of emphysema on computed tomography (CT)) in IPF patients did not independently predict mortality once summed visual lobar CT extents of emphysema and interstitial lung disease (ILD) had been considered. Put another way, survival in CPFE patients was the same as for IPF patients without emphysema, once the total extents of emphysema and fibrosis on CT were considered. The findings suggested that there was no additional synergistic impact on mortality when both disease patterns (emphysema and ILD) co-existed. Past analyses of CPFE populations have shown conflicting results regarding the impact of CPFE on mortality [3,5-11] and may relate to heterogeneous study populations, varied CPFE inclusion criteria, and inconsistent adjustment for disease severity in mortality models [12]. The aim of the current study was to confirm our earlier study findings [4] that a CPFE phenotype has no independent mortality effect beyond that described by emphysema and ILD extent. The question was evaluated using various definitions of CPFE that have been considered in the literature, with results run on independent validation datasets.

We evaluated two separate cohorts of IPF patients diagnosed by a multidisciplinary team. Cohort 1: 220 patients from two centres in Turkey and Italy, 102 deaths observed; cohort 2: 310 patients from two centres in the Netherlands and England, and from the Australian IPF Registry, 169 deaths observed. CT extents of emphysema and ILD were separately scored, averaged across the lobes and then summed to develop a total lung percentage for both patterns as previously described [4]. We also performed a sub analysis in IPF patients that fulfilled drug trial inclusion criteria $\left(D_{\text {LCO }}>30 \%\right.$ predicted and FVC $>50 \%$ predicted) in cohort 1 ( $\mathrm{n}=150,57$ deaths observed) and cohort 2 ( $\mathrm{n}=239,117$ deaths observed). The median and interquartile ranges of emphysema extent were $4.17 \%$ and $11.67 \%$ in cohort $1 ; 2.92 \%$ and $8.33 \%$ in cohort 2 . In the populations qualifying for drug trials: median and interquartile ranges of emphysema extent were $3.33 \%$ and $10.00 \%$ in cohort $1 ; 2.50 \%$ and $7.50 \%$ in cohort 2 .

In each cohort, multivariable mixed-effects Cox regression models were used to evaluate whether the CPFE phenotype had any impact on outcome after considering the sum of visual CT extents of ILD and emphysema: VILDemph. To ensure that VILDemph and the various expressions of CPFE could be applied in the same model, we tested for collinearity using univariable linear regression. No strong collinearity was

Shareable abstract (@ERSpublications)

In patients with combined pulmonary fibrosis and emphysema, emphysema and fibrosis do not have a synergistic effect that results in worsened survival when compared to IPF patients without emphysema https://bit.ly/35EJMo6

Cite this article as: Zhao A, Gudmundsson E, Mogulkoc N, et al. Mortality in combined pulmonary fibrosis and emphysema patients is determined by the sum of pulmonary fibrosis and emphysema. ERJ Open Res 2021; 7: 00316-2021 [DOI: 10.1183/23120541.00316-2021]. 
shown between VILDemph and the various expressions of CPFE (maximum $\mathrm{R}^{2}=0.36$ ). All mortality models were adjusted for patient age, sex, smoking status (never versus ever) and antifibrotic use (never versus ever). Models were repeated evaluating $D_{\mathrm{LCO}}$ instead of VILDemph as a distinct functional measure of disease severity, thereby complimenting the models where a morphological measure of disease severity had been used (VILDemph). Different centres/countries within each cohort were modelled as multilevel with random effects between centres/countries (with a random intercept per centre/country). To encompass the breadth of published definitions of the CPFE phenotype [12], the CPFE phenotype was separately characterised as a binary emphysema variable in multivariable mixed-effects Cox regression models using four different emphysema thresholds $(0 \%, 5 \%, 10 \%$ or $15 \%$ emphysema). The concordance index (C-index) was used to compare the predictive performance of the Cox models. Bootstrapping with 500 replications was used in the estimation of the C-index. p-values $<0.05$ were regarded as statistically significant. All analyses were implemented by R Studio.

Our results demonstrated that in both IPF cohorts, the CPFE phenotype did not independently predict mortality once summed extents of ILD and emphysema were considered in multivariable models (table 1). The results were maintained when patients fulfilling drug trial entry criteria were sub analysed in both cohorts. Results remained unchanged when the models examined baseline $D_{\text {LCO }}$ instead of VILDemph to adjust for disease severity. 181 (82\%) out of 220 patients in cohort 1, and 266 (86\%) out of 310 patients in cohort 2 had baseline $D_{\text {LCO }}$ values, whilst all patients in the drug trial population had baseline $D_{\text {LCo }}$ values.

Our study confirms that mortality in patients with CPFE is explained by the sum of its two disease processes: the extents of fibrosis and emphysema. CPFE does not appear to manifest a malignant phenotype where survival is worse than that expected from the combination of two bad disease processes. Accordingly, once you consider emphysema and ILD patterns on CT, survival in CPFE is no different to survival in IPF patients without emphysema. The results were maintained when all of the different definitions of CPFE were separately analysed in both study cohorts and the smaller subsets of patients that would be included in drug trials.

A limitation of the study by ЈАСов et al. [4] was that the extent of emphysema in the cohort was relatively limited, with 11\% (30 out of 272) patients having >15\% emphysema extent (a threshold above which

TABLE 1 Multivariable mixed-effects Cox proportional hazards regression models in two cohorts of idiopathic pulmonary fibrosis (IPF) patients

\begin{tabular}{|c|c|c|c|c|c|}
\hline Disease severity and emphysema variables in the models & Patients for emphysema thresholds & C-index & Hazard ratio & p-value & $95 \% \mathrm{Cl}$ \\
\hline \multicolumn{6}{|l|}{ Cohort 1} \\
\hline VILDemph & Emphysema $=0 \%: n=79$ & \multirow{2}{*}{0.76} & 1.05 & $<0.0001$ & $(1.04-1.06)$ \\
\hline Binary visual emphysema (threshold: $0 \%$ ) & Emphysema $>0 \%: n=141$ & & 1.03 & 0.931 & $(0.55-1.94)$ \\
\hline VILDemph & Emphysema $\leqslant 5 \%: n=123$ & \multirow{2}{*}{0.76} & 1.05 & $<0.0001$ & $(1.04-1.07)$ \\
\hline Binary visual emphysema (threshold: 5\%) & Emphysema $>5 \%: n=97$ & & 0.62 & 0.090 & $(0.35-1.08)$ \\
\hline VILDemph & Emphysema $\leqslant 10 \%: n=155$ & \multirow{2}{*}{0.76} & 1.05 & $<0.0001$ & $(1.04-1.07)$ \\
\hline Binary visual emphysema (threshold: $10 \%$ ) & Emphysema $>10 \%: n=65$ & & 0.75 & 0.273 & $(0.44-1.26)$ \\
\hline VILDemph & Emphysema $\leqslant 15 \%: \mathrm{n}=179$ & \multirow{2}{*}{0.76} & 1.05 & $<0.0001$ & $(1.04-1.07)$ \\
\hline Binary visual emphysema (threshold: 15\%) & Emphysema $>15 \%: n=41$ & & 0.71 & 0.244 & $(0.39-1.27)$ \\
\hline \multicolumn{6}{|l|}{ Cohort 2} \\
\hline VILDemph & Emphysema $=0 \%: n=126$ & \multirow{2}{*}{0.70} & 1.03 & $<0.0001$ & $(1.02-1.04)$ \\
\hline Binary visual emphysema (threshold: $0 \%$ ) & Emphysema $>0 \%: \mathrm{n}=184$ & & 0.81 & 0.234 & $(0.58-1.14)$ \\
\hline VILDemph & Emphysema $\leqslant 5 \%$ : $n=202$ & \multirow{2}{*}{0.71} & 1.03 & $<0.0001$ & $(1.02-1.04)$ \\
\hline Binary visual emphysema (threshold: 5\%) & Emphysema $>5 \%: n=108$ & & 0.79 & 0.231 & $(0.55-1.16)$ \\
\hline VILDemph & Emphysema $\leqslant 10 \%: \mathrm{n}=248$ & \multirow{2}{*}{0.70} & 1.03 & $<0.0001$ & $(1.02-1.04)$ \\
\hline Binary visual emphysema (threshold: $10 \%$ ) & Emphysema $>10 \%: n=62$ & & 0.76 & 0.225 & $(0.49-1.18)$ \\
\hline VILDemph & Emphysema $\leqslant 15 \%: n=271$ & \multirow{2}{*}{0.70} & 1.03 & $<0.0001$ & $(1.02-1.04)$ \\
\hline Binary visual emphysema (threshold: $15 \%$ ) & Emphysema $>15 \%: \mathrm{n}=39$ & & 0.68 & 0.160 & $(0.40-1.16)$ \\
\hline
\end{tabular}

To evaluate whether the combined pulmonary fibrosis and emphysema (CPFE) phenotype had an additive impact on outcome of IPF patients after adjusting for patient age, sex, smoking status (never versus ever), antifibrotic use (never versus ever) and baseline disease severity, multivariable mixed-effects Cox regression models were used to analyse two independent cohorts of IPF patients. Separate centres/countries within cohort 1 and cohort 2 were modelled as multilevel with random effects between centres/countries (a random intercept per centre/country). Baseline disease severity was quantified as the sum of average lobar visual CT extents of emphysema and ILD: VILDemph. The CPFE phenotype was separately characterised by a binary visual emphysema variable using thresholds of emphysema extent including: $0 \%, 5 \%, 10 \%$ or $15 \%$, which were reported in various definitions of CPFE in the literature. The impact of the four binary visual emphysema thresholds were separately analysed in multivariable models. C-index: concordance index. 
emphysema has been associated with significantly reduced FVC decline [13]). The proportion of patients with emphysema $>15 \%$ was higher in the current study populations, 41 (19\%) out of 220 patients in cohort 1 and 39 (13\%) out of 310 patients in cohort 2. There were also very few patients in whom emphysema extent was greater than fibrosis extent. Only five (2\%) out of 220 patients in cohort 1 and 13 (4\%) out of 310 patients had more emphysema than fibrosis. A recent CPFE study considered patients in whom emphysema was more extensive than ILD on CT [14]. Repeating our analyses with a CPFE population defined in this way would be important to confirm our findings. Yet powering such a study in IPF patients will be extremely challenging.

In summary, we have validated findings across independent datasets confirming that in CPFE patients mortality is explained by the sum of emphysema and fibrosis extents. We have demonstrated that in CPFE patients, emphysema and fibrosis do not have a synergistic effect resulting in a malignant disease phenotype. CPFE patients and IPF patients without emphysema have indistinguishable mortality once the extents of emphysema and ILD on CT have been considered.

An Zhao ${ }^{1}$, Eyjolfur Gudmundsson ${ }^{1}$, Nesrin Mogulkoc ${ }^{2}$, Mark G. Jones $\oplus^{3}$, Coline van Moorsel ${ }^{4}$, Tamera J. Corte ${ }^{5}$, Chiara Romei ${ }^{6}$, Recep Savas ${ }^{7}$, Christopher J. Brereton ${ }^{3}$, Hendrik W. van Es ${ }^{4}$, Helen $\mathrm{Jo}^{5}$, Annalisa De Liperi ${ }^{6}$, Omer Unat ${ }^{2}$, Katarina Pontoppidan ${ }^{3}$, Frouke van Beek ${ }^{4}$, Marcel Veltkamp ${ }^{8,9}$, Peter Hopkins ${ }^{10}$, Yuben Moodley ${ }^{11,12}$, Alessandro Taliani ${ }^{6}$, Laura Tavanti ${ }^{13}$, Bahareh Gholipour ${ }^{14}$, Arjun Nair ${ }^{14}$, Sam Janes $\oplus^{15}$, lain Stewart $\oplus^{16}$, David Barber ${ }^{17}$, Daniel C. Alexander ${ }^{1}$, Athol U. Wells ${ }^{18,19}$ and Joseph Jacob $\oplus^{1,15}$

${ }^{1}$ Centre for Medical Image Computing, UCL, London, UK. ${ }^{2}$ Dept of Respiratory Medicine, Ege University Hospital, Izmir, Turkey. ${ }^{3}$ Clinical and Experimental Sciences, Faculty of Medicine, University of Southampton and NIHR Southampton Biomedical Research Centre, University Hospital Southampton, Southampton, UK. ${ }^{4}$ Dept of Radiology, St Antonius Hospital, Nieuwegein, The Netherlands. ${ }^{5}$ Dept of Respiratory Medicine, Royal Prince Alfred Hospital, Camperdown, Australia. ${ }^{6}$ Dept of Radiology, Pisa University Hospital, Pisa, Italy. ${ }^{7}$ Dept of Radiology, Ege University Hospital, Izmir, Turkey. ${ }^{8}$ Dept of Pulmonology, Interstitial Lung Diseases Center of Excellence, St Antonius Hospital, Nieuwegein, The Netherlands. ${ }^{9}$ Division of Heart and Lungs, University Medical Center, Utrecht, The Netherlands. ${ }^{10}$ Queensland Centre for Pulmonary Transplantation and Vascular Disease, The Prince Charles Hospital, Chermside, Australia. ${ }^{11}$ School of Medicine and Pharmacology, University Western Australia, Perth, Australia. ${ }^{12}$ Fiona Stanley Hospital, Perth, Australia. ${ }^{13}$ Cardiovascular and Thoracic Dept, Pisa University Hospital, Pisa, Italy. ${ }^{14}$ Dept of Radiology, University College London Hospitals NHS Foundation Trust, London, UK. ${ }^{15}$ Lungs for Living Research Centre, UCL, London, UK. ${ }^{16}$ National Heart and Lung Institute, Imperial College London, London, UK. ${ }^{17}$ Centre for Artificial Intelligence, UCL, London, UK. ${ }^{18}$ Dept of Respiratory Medicine, Royal Brompton Hospital, London, UK. ${ }^{19}$ Imperial College London, London, UK.

Corresponding author: Joseph Jacob (j.jacob@ucl.ac.uk)

Submitted article, peer reviewed.

Conflict of interest: A. Zhao has nothing to disclose. E. Gudmundsson has nothing to disclose. N. Mogulkoc has nothing to disclose. M.G. Jones has nothing to disclose. C. van Moorsel has nothing to disclose. T.J. Corte reports personal fees from Ad Alta, grants and personal fees from Boehringer Ingelheim and Bristol Myers Squibb, personal fees from Promedior, grants and personal fees from Roche, and grants from Actelion, Avalyn Pharma, Biogen and Galapagos, outside the submitted work. C. Romei has nothing to disclose. R. Savas has nothing to disclose. C.J. Brereton has nothing to disclose. H.W. van Es has nothing to disclose. H. Jo has nothing to disclose. A. De Liperi has nothing to disclose. O. Unat has nothing to disclose. K. Pontoppidan has nothing to disclose. F. van Beek has nothing to disclose. M. Veltkamp has nothing to disclose. P. Hopkins has nothing to disclose. Y. Moodley has nothing to disclose. A. Taliani has nothing to disclose. L. Tavanti has nothing to disclose. B. Gholipour has nothing to disclose. A. Nair reports a proportion of their permanent employment at UCL Hospital is funded by the Biomedical Research Centre, and nonfinancial support for an advisory board from Aidence BV, the Netherlands, outside the submitted work. S. Janes reports personal fees and nonfinancial support from AstraZeneca, personal fees from Bard1 Bioscience, Achilles Therapeutics and Jansen, nonfinancial support from Takeda, and grants from GRAIL Inc., GlaxoSmithKline plc and from Owlstone, outside the submitted work. I. Stewart has nothing to disclose. D. Barber has nothing to disclose. D.C. Alexander has nothing to disclose. A.U. Wells reports personal fees and nonfinancial support from Boehringer Ingelheim, Bayer and Roche 
Pharmaceuticals, and personal fees from Blade, outside the submitted work. J. Jacob reports personal fees from Boehringer Ingelheim and Roche, grants and personal fees from GlaxoSmithKline, personal fees from NHSX, and grants from the Wellcome Trust, outside the submitted work.

Support statement: This study was supported by Wellcome Trust grant 209553/Z/17/Z and the UCLH Biomedical Research Centre. Funding information for this article has been deposited with the Crossref Funder Registry.

\section{References}

1 King CS, Nathan SD. Idiopathic pulmonary fibrosis: effects and optimal management of comorbidities. Lancet Respir Med 2017; 5: 72-84.

2 Lin H, Jiang S. Combined pulmonary fibrosis and emphysema (CPFE): an entity different from emphysema or pulmonary fibrosis alone. J Thorac Dis 2015; 7: 767-779.

3 Cottin V, Nunes H, Brillet PY, et al. Combined pulmonary fibrosis and emphysema: a distinct underrecognised entity. Eur Respir J 2005; 26: 586-593.

4 Jacob J, Bartholmai BJ, Rajagopalan S, et al. Functional and prognostic effects when emphysema complicates idiopathic pulmonary fibrosis. Eur Respir J 2017; 50: 1700379.

5 Mejía M, Carrillo G, Rojas-Serrano J, et al. Idiopathic pulmonary fibrosis and emphysema: decreased survival associated with severe pulmonary arterial hypertension. Chest 2009; 136: 10-15.

6 Kurashima K, Takayanagi N, Tsuchiya N, et al. The effect of emphysema on lung function and survival in patients with idiopathic pulmonary fibrosis. Respirology 2010; 15: 843-848.

7 Jankowich MD, Rounds S. Combined pulmonary fibrosis and emphysema alters physiology but has similar mortality to pulmonary fibrosis without emphysema. Lung 2010; 188: 365-373.

8 Ryerson CJ, Hartman T, Elicker BM, et al. Clinical features and outcomes in combined pulmonary fibrosis and emphysema in idiopathic pulmonary fibrosis. Chest 2013; 144: 234-240.

9 Sugino K, Ishida F, Kikuchi N, et al. Comparison of clinical characteristics and prognostic factors of combined pulmonary fibrosis and emphysema versus idiopathic pulmonary fibrosis alone. Respirology 2014; 19: 239-245.

10 Zhang L, Zhang C, Dong F, et al. Combined pulmonary fibrosis and emphysema: a retrospective analysis of clinical characteristics, treatment and prognosis. BMC Pulm Med 2016; 16: 137.

11 Jiang C-G, Fu Q, Zheng C-M. Prognosis of combined pulmonary fibrosis and emphysema: comparison with idiopathic pulmonary fibrosis alone. Ther Adv Respir Dis 2019; 13: 1753466619888119.

12 Wong AW, Liang J, Cottin V, et al. Diagnostic features in combined pulmonary fibrosis and emphysema: a systematic review. Ann Am Thorac Soc 2020; 17: 1333-1336.

13 Cottin V, Hansell DM, Sverzellati N, et al. Effect of emphysema extent on serial lung function in patients with idiopathic pulmonary fibrosis. Am J Respir Crit Care Med 2017; 196: 1162-1171.

14 Zantah M, Dotan Y, Dass C, et al. Acute exacerbations of COPD versus IPF in patients with combined pulmonary fibrosis and emphysema. Respir Res 2020; 21: 164. 